\title{
Self-Interference Channel Characterization for Reconfigurable Antenna Based Systems
}

\author{
Sergey Shaboyan ${ }^{1}$, Alireza S. Behbahani ${ }^{1}$, and Ahmed M. Eltawil ${ }^{1,2}$ \\ ${ }^{1}$ Department of Electrical Engineering and Computer Science, University of California, Irvine, California, USA. \\ ${ }^{2}$ Computer, Electrical and Mathematical Science and Engineering Division, \\ King Abdullah University of Science and Technology (KAUST), Thuwal, Saudi Arabia. \\ Emails :\{sshaboya,sshahanb\}@uci.edu, aeltawil@ieee.org
}

\begin{abstract}
This paper presents experimental characterization of the self-interference (SI) channel for In-Band Full-Duplex (IBFD) communication systems that employ beam steering, Multi reconfigurable antennas (MRA), for passive SI suppression. First, we describe a system model of a full-duplex base station while highlighting channel impairments associated with each signal component. We present the SI channel power delay profiles measured by IBFD system operating on $5 \mathrm{MHz}, 10 \mathrm{MHz}$ and $20 \mathrm{MHz}$ bandwidths. We compare the measurements for beam patterns that result in line of sight suppression (and non-suppression) in both static and dynamic environments. We then statistically model the SI channel by performing probability distribution fitting to SI channel data, which is collected in dynamic environment, under high and low SI suppression conditions provided by MRA. Finally, candidate probability distribution models are compared using goodness of fit test.
\end{abstract}

Keywords-In Band Full-Duplex; Channel; Self-Interference.

\section{INTRODUCTION}

W IRELESS networks are demanding high data rate to accommodate requests from various applications. InBand Full-Duplex (IBFD) communication is an emerging solution which is rapidly becoming accepted as a means of improving spectral efficiency in order to efficiently utilize limited frequency spectrum. In the IBFD communication systems, a wireless terminal is allowed to transmit and receive simultaneously in the same frequency band. The key challenge in FD wireless communications is the existence of strong selfinterference (SI) that must be suppressed to a sufficiently low level in order to have proper receiver operation with negligible performance degradation. In general, SI cancellation schemes can be applied across spatial, analog, and digital domains. In order to accurately reconstruct the received SI based on the known transmitted signal, an accurate estimation of the SI channel is required. Accurate SI channel estimation obviously requires a clear understanding of SI channel propagation characteristics and modeling.

Traditional radio channels are well understood and characterized with mature channel models when the transmitter and receiver are isolated and separated. However, only few works have investigated the SI channel and its effect on system performance. The authors in [1], consider SI channel propagation characteristics for a two dipole antenna in an indoor environment when operating at $2.6 \mathrm{GHz}$ with $200 \mathrm{MHz}$ bandwidth. The statistical model of path loss factor and Ricean $\mathrm{K}$-factor for different antenna separation was proposed and discussed. Indoor mobile single-input single-output (SISO) SI channel propagation characteristics of a shared single omnidirectional dipole antenna with circulator were investigated in [2]. The measurements indicate that the corresponding SI channel power delay profile (PDP) has three components: the leakage path, the reflection due to antenna port mismatch, and multipath fading due to surrounding environment. The space multipath component has been modeled by a power-law decay with a log-normal variation. The coherence bandwidth of the SISO SI channel for both the shared and the separate antenna architectures of the IBFD for 3-10 GHz ultra-wideband (UWB) have been studied in [3]. One of the key results is that the coherence bandwidth of the SI channel is found to be varying between 1 and $4 \mathrm{MHz}$. In [4], the wideband SI channel for outdoor-to-indoor relay was characterized with $300 \mathrm{MHz}$ bandwidth centered at $2.6 \mathrm{GHz}$. Their measurements indicate that the SI relay channel can be modelled as a single decaying exponential function with specular components, and the small scale fading of each tap was modeled by Rician distribution. The authors in [5] present a measurement based study of the capabilities and limitations of three key mechanisms for passive self-interference suppression: directional isolation, absorptive shielding, and cross polarization. The study demonstrates that more than $70 \mathrm{~dB}$ of passive suppression can be achieved in certain environments. They found that environmental reflections limit cancellation performance where passive SI suppression can be achieved. In addition, higher passive SI suppression generally results in serious frequency selectivity of the SI channel.

In this paper, we perform channel characterization of selfinterference IBFD-OFDM systems that use beam steering, multi-reconfigurable antenna (MRA) as a passive SI suppression mechanism. Specifically, the paper presents the following contributions:

- Measurements of SI channel are presented that are collected using IBFD-OFDM software-defined radio (SDR) platform under scenarios of high and low suppression levels provided by MRA, static/dynamic environments and system bandwidth of $5 \mathrm{MHz}, 10 \mathrm{MHz}$ $20 \mathrm{MHz}$.

- Comparison between measured channel delay profiles along with frequency responses is presented highlighting the significant differences and their impact on complexity of SI suppression mechanisms.

- Statistical modeling of SI channel is performed by fitting probability distribution to the collected data and comparing the results by conducting goodness of fit test.

The remainder of the paper is organized as follows. In Section II, MRA based IBFD system is described and the received level of SI along with its impact on system performance depending on selected MRA pattern are presented. In 
Section III, experimental setup for SI channel measurements is described. SI channel measurements under different scenarios are presented and compared in Section IV. Channel characterization based on statistical modeling is presented in Section $\mathrm{V}$. The paper is concluded in Section VI.

1) Notation: We use $(*)$ to denote convolution, $(.)^{*}$ to denote conjugate. Time domain variables are represented as lowercase letters, while frequency domain variables use uppercase.

\section{Full DupleX System}

A wireless system operating in full-duplex mode is illustrated in figure 1 . The system consists of base station receiving from a remote node $\mathrm{N} 2$, at the same time transmitting to another node in the same frequency band. Signals transmitted by the remote node to the base station are referred to as signal of interest (SOI), and the transmitted signal by the base station causes SI. All the nodes employ Orthogonal Frequency Division Multiplexing (OFDM) with $N_{F F T}$ subcarriers, therefore the $i^{\text {th }}$ block of received signal by the base station in the frequency domain can be modeled as

$$
R_{i}(k)=S_{i}^{t x}(k) H_{i}^{s o i}(k)+Y_{i}^{t x}(k) H_{i}^{s i}(k)+W_{i}(k)
$$

where $S^{t x}(k)$ and $Y^{t x}(k)$ are SOI and SI components of the transmitted signal. $H_{i}^{s o i}(k)$ and $H_{i}^{s i}(k)$ are channels frequency responses of SOI and SI respectively. $W(k)$ is Additive White Gaussian Noise (AWGN).

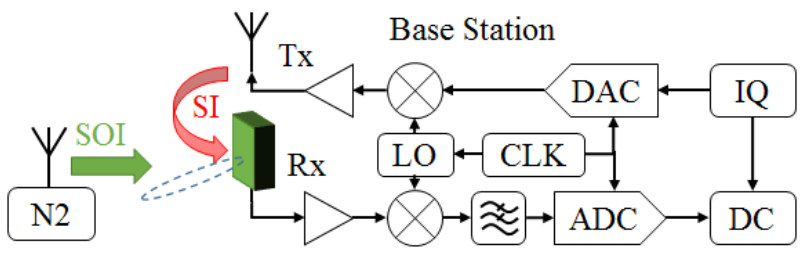

Fig. 1: Block diagram of full duplex node.

From Eq. (1) it is clear that SI must be suppressed in order to achieve satisfactory Signal to Interference and Noise Ratio (SINR) for the received signal. Multiple techniques for suppressing SI exist across multiple domains such as propagation, analog and digital. The base station is equipped with a multi-reconfigurable antenna (MRA), which provides significant passive suppression of self-interference in the propagation domain, meanwhile preventing saturation of $\mathrm{Rx}$ fontend. It is a single antenna element, equipped with rectangular patch segments, that can either be connected together with low loss switches or can be left floating. This causes the antenna geometry to change consequently changing its radiation pattern dynamically. Figure $2 \mathrm{~b}$ shows a 3D shape of a selected radiation pattern, and Figure 2 a illustrates $2 \mathrm{D}$ radiation patterns for different switch combinations, plotted on top of each other to highlight their differences. Aiming for maximum passive SI suppression, the search algorithm selects the radiation pattern that achieves the weakest $H^{s i}(k)$.

To achieve active SI suppression, digital cancellation is used as a second stage after MRA. At this step the receiver uses the knowledge of a local copy of the transmitted signal to

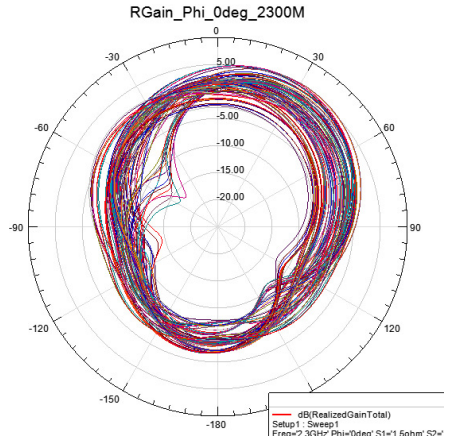

(a) 2D Radiation Pattern

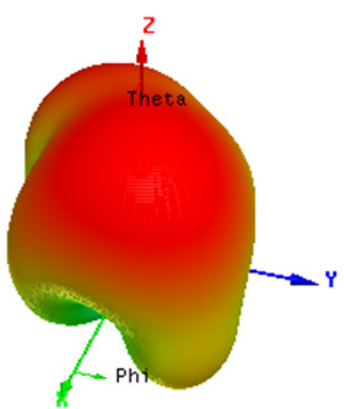

(b) 3D Radiation Pattern
Fig. 2: MRA Radiation Pattern

perform subtraction from the received signal, which contains the superposition of SOI and SI. While subtracting a known signal (SI known transmit signal) seems straightforward, numerous analog components in the Tx chain distort the signal both linearly and non-linearly, complicating the cancellation process. Furthermore, noise mechanisms associated with the phase locked loop and receiver components further complicate the problem. Therefore naively subtracting the baseband signal from the received signal without accounting for all the distortions results in limited cancellation performance. Assuming perfect synchronization between the nodes the received signal after digital cancellation can be expressed as

$D_{i}(k)=S_{i}^{t x}(k) H_{i}^{s o i}(k)+Y_{i}^{t x}(k)\left(H_{i}^{s i}(k)-\hat{H}_{i}^{s i}(k)\right)+W_{i}(k)$

where $\hat{H}^{s i}(k)$ is the estimated SI channel at digital cancellation step. In this work, least square (LS) estimation method is used for estimating SI channel at the receiving node.

$$
\hat{H}_{i}^{s i}(k)=\frac{P_{i}^{s i}(k)}{Y_{i}^{t x}(k)}=\frac{Y_{i}^{t x}(k) H_{i}^{s i}(k)+W_{i}(k)}{Y_{i}^{t x}(k)},
$$

where $P_{i}^{s i}(k)$ is the local copy of $i^{t h}$ block of SI preamble. From (2) it is obvious that the interference term diminishes as the difference between the actual and estimated SI channels decreases. Considering finite accuracy of SI channel estimation, the received signal after digital cancellation can be expressed as

$$
D_{i}(k)=S_{i}^{t x}(k) H_{i}^{s o i}(k)+W_{i}^{s i}(k)+W_{i}(k)
$$

where $W^{s i}(k)$ is the residual uncompensated self-interference, and is due to SI channel estimation error [6].

\section{EXPERIMENTAL SySTEM SETUP}

A complete full-duplex experimental system is constructed using the Universal Software Radio Peripheral (USRP) SDR platform. Each USRP contains a Radio Frequency (RF) transceiver and a Field Programmable Gate Array (FPGA). All USRPs are connected to a host PC through a Gigabit Ethernet connection. The baseband signal processing is performed over the host PC. The baseband signals are streamed to/from the USRPs at a rate of $25 \mathrm{M}$ sample/sec. The RF transceivers are then used for real time signal transmission and reception.

As shown in Figure 3, the base Station is equipped with one transmit and one receive antenna situated $10 \mathrm{~cm}$ apart 
from each other. Node 2 uses only one antenna. A monopole omni-directional antenna is used as transmit antenna, while a reconfigurable directional antenna is used as a receive antenna. Both transmit and receive antennas have the same antenna polarization. The reconfigurable antenna has a total of 4096 different possible radiation patterns. A successful pattern, is a pattern that maximizes the SOI signal and minimizes the SI signal. The pattern selection is performed through a 12-line digital control cable driven from an FPGA on a Zedboard. The timing of all USRPs and the FPGA that drive the antenna radiation selection are aligned with one reference Pulse Per Second (PPS) signal. All the signals are transmitted according to parameters listed in Table I.

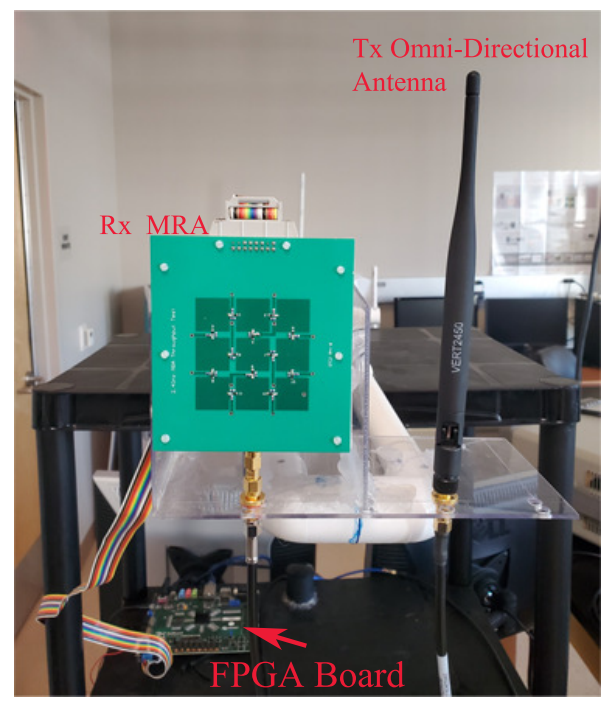

Fig. 3: Tx and Rx antenna setup on FD base station

\begin{tabular}{|c|c|c|c|}
\hline OFDM Parameters & Value & Signal Parameters & Value \\
\hline Number of OFDM Subcarriers & 64 & Carrier Frequency & $2.5 \mathrm{GHz}$ \\
Number of Data Subcarriers & 44 & Sampling Rate & $5-20 \mathrm{MHz}$ \\
Cyclic Prefix (CP) & $3.2 \mu \mathrm{s}$ & Tx Power & $10 \mathrm{dBm}$ \\
Symbol Duration (CP+FFT) & $16 \mu \mathrm{s}$ & Rx SOI Power & $-65 \mathrm{dBm}$ \\
Long Training Duration & $32 \mu \mathrm{s}$ & Rx SI Power SP & $-60 \mathrm{dBm}$ \\
Short Training Duration & $16 \mu \mathrm{s}$ & Rx SI Power NSP & $-25 \mathrm{dBm}$ \\
\hline
\end{tabular}

TABLE I: Experimental system parameters

\section{Channel Measurements and Comparison}

To capture the SI channel characteristics depending on selected MRA pattern, the received signal i probed in the digital domain (after ADC) before the digital cancellation step shown in Figure 1. Since the MRA has 4096 different patterns, each selected pattern can be viewed as a unique channel. To simplify the study we examine the SI channel when a suppressing pattern (SP) is selected, providing SI suppression of at least $60 \mathrm{~dB}$, and a non-suppressing pattern (NSP) is selected, in which case SI suppression is only $20 \mathrm{~dB}$ due to antenna separation. The channel measurements are performed by sending a test signal and measuring the channel response at the receiver. To capture the SI channel effects on narrow-band and wideband operations, the channel measurement are acquired using a system bandwidth of $5 \mathrm{MHz}, 10 \mathrm{MHz}$ and 20MHz. The test signal is generated using continues transmission of consecutive packets, where each packet is constructed according to a modified WiFi frame structure with non-overlapping training preamble enabling the receiver to estimate the channels of individual signal components of SI and SOI in FD mode. [7].

\section{A. NSP Channel Measurements}

SI channel measurements were collected in the lab during static and dynamic environments. Channel dynamics were introduced by manually moving a reflector object with $1 \mathrm{~m}^{2}$ flat surface area approximately $2 m$ away from the base station. Figure 4 illustrates the power delay profile of SI channel under the scenario when a non-suppressing pattern is selected by MRA. From the figure, it is clear that due to the low suppression of SI there exist one strong channel path that dominates regardless of the transmitted signal bandwidth and existence of surrounding reflecting objects. The channel power delay profile was not affected by the introduced dynamics due to the presence of strong SI signal transmitted from a source $10 \mathrm{~cm}$ away, which is orders of magnitude larger than the reflections from object located $2 \mathrm{~m}$ away.

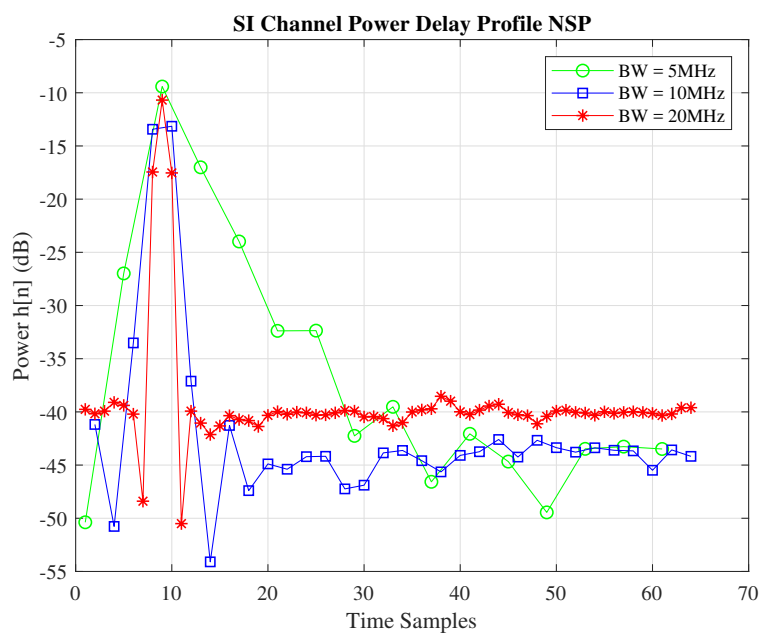

Fig. 4: SI channel delay profile in static and dynamic environments.

\section{B. SP Channel Measurements}

In the case when a suppressing pattern is selected by MRA, an additional SI suppression of $35 \mathrm{~dB}$ is observed, which weakens the direct sight of SI channel, effectively making the SI reflections from indirect sight significant. Figure 5 illustrates the SI channel delay profile when SP is selected in static environment. Due to the minimal changes in environment, the power variations of the channel taps are minimal. As a result the channel taps with dominant powers do not experience much variation throughout the experiment. For narrow-band system, the SI channel can be approximated as having one dominant tap, whereas for larger bandwidth, such as $10 \mathrm{MHz}$ and $20 \mathrm{MHz}$ multi-path components come into the picture. The presence of significant multi-path creates additional channel taps and consequently frequency selectivity, which can be seen in the channel frequency response plotted in Figure 6.

Figure 7 illustrates the SI channel power delay profile in a dynamic environment. In this case, due to the moving reflecting 


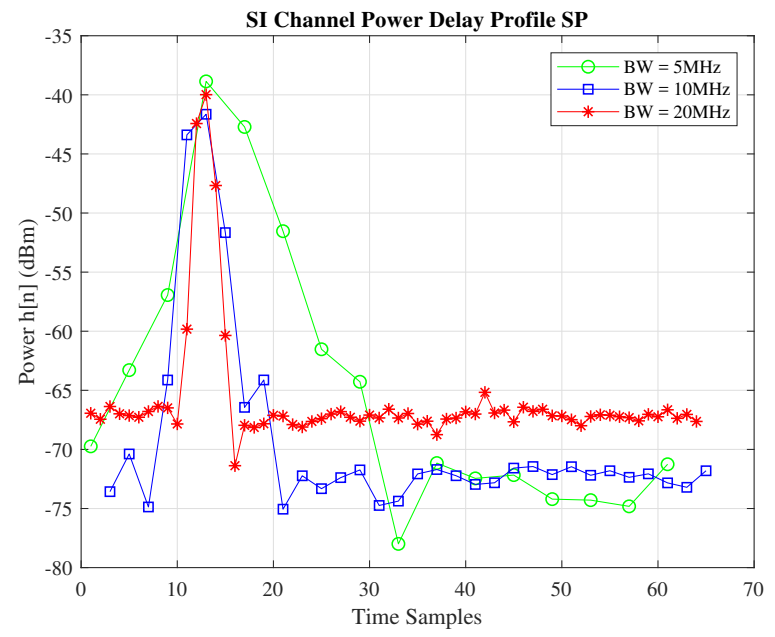

Fig. 5: SI channel delay profile in static environment.

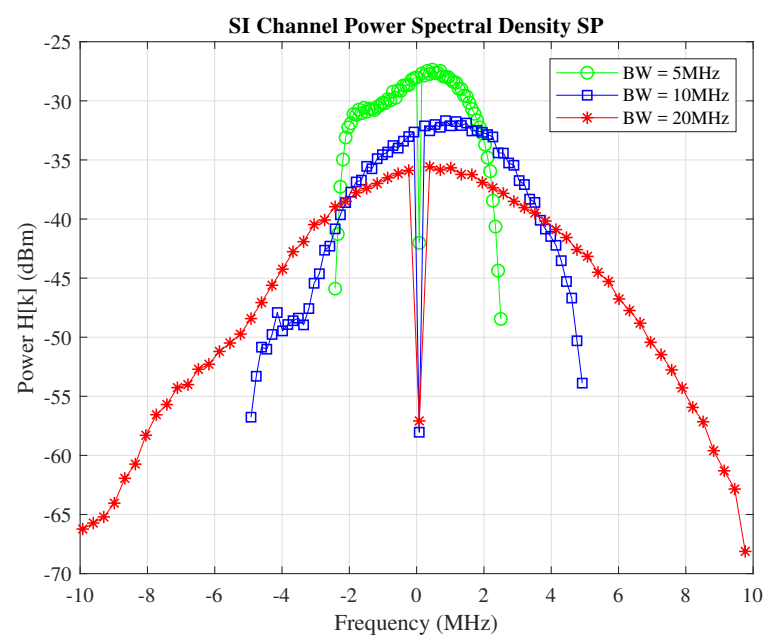

Fig. 6: SI channel frequency profile in static environment.

object, even the dominant SI channel taps are experiencing large power level variations over time. As opposed to the NSP case, the introduced environment dynamics become more significant with larger SI suppression. Comparing channel delay profiles of static and dynamic environments (Figure 7 and Figure 5), it is obvious that dynamic SI channel has larger delay spread, introducing frequency selectivity even for narrow-band transmission. By comparing the estimated frequency response of static and dynamic channels (Figure 6 and Figure 8), it is obvious that frequency selectivity in dynamic channel is noticeably increased for all three bandwidths.

\section{Discussion}

The SI channel characteristics directly impact the SI suppression capability and the complexity of suppressing mechanisms. Initially SI suppression is small, and there is a single dominant SI path, while multi-path are insignificant (Figure 4). As the dominant path is being suppressed, some reflections become significant requiring additional suppression stages especially for wide-band transmission (Figure 5). In addition,

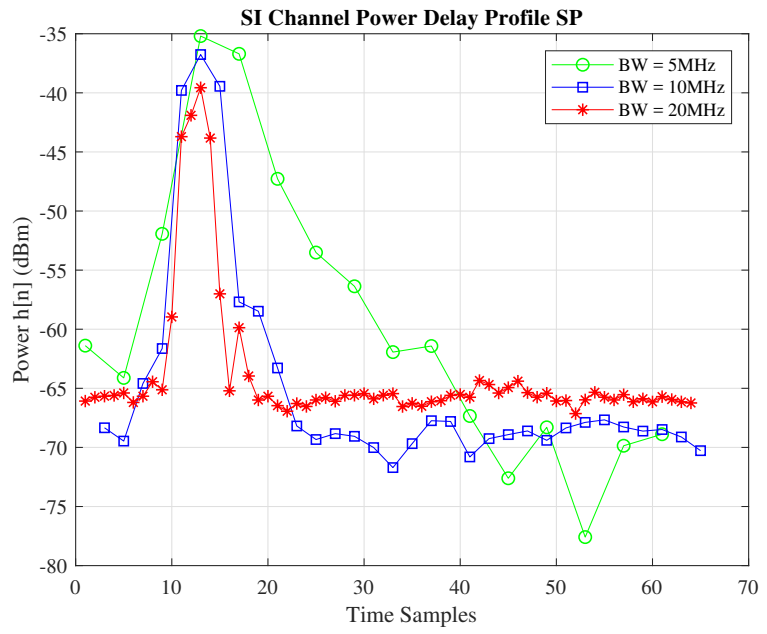

Fig. 7: SI channel delay profile in dynamic environment.

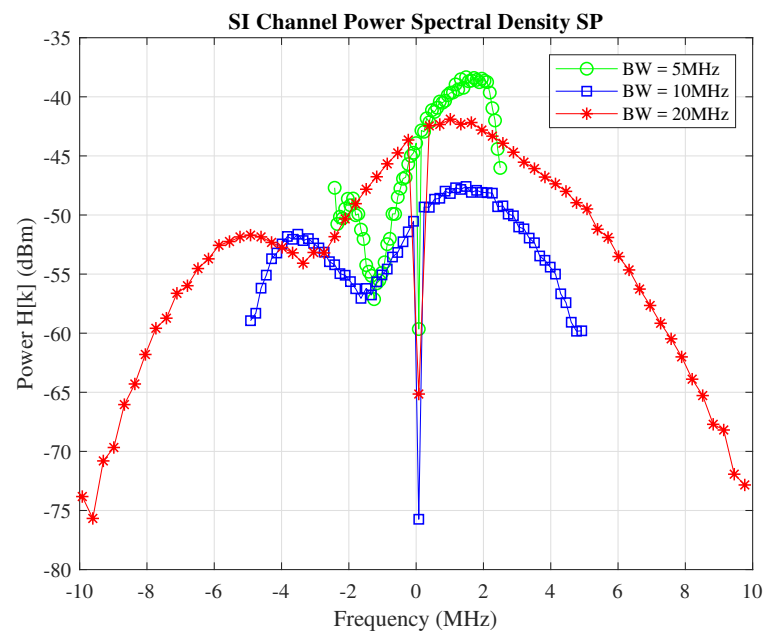

Fig. 8: SI channel frequency profile in dynamic environment.

if a dynamic environment is introduced, then suppression of SI becomes even more challenging with increased delay spread and varying power levels of channel delay taps as shown in Figure 7.

\section{SI CHANNEL CHARACTERIZATION}

The channel model illustrated in Figure 1 represents both SOI and SI channels. SOI channel can be modeled as Rayleigh fading channel, considering the absence of Line-Of-Sight (LOS) component due to the location of Node2 [8].

In order to properly choose a realistic model for SI channel, we conducted a brief statistical study of the SI channel for the scenarios when SP and NSP are selected by MRA. For each of the two patterns, over 5000 SI channel measurements are collected. We then performed fitting of Rayleigh and Rician probability density functions (PDF) into collected data, using maximum likelihood estimation (MLE) of parameters. To conduct a fair comparison between the candidate models we have used two parameter Rayleigh distribution, where an 


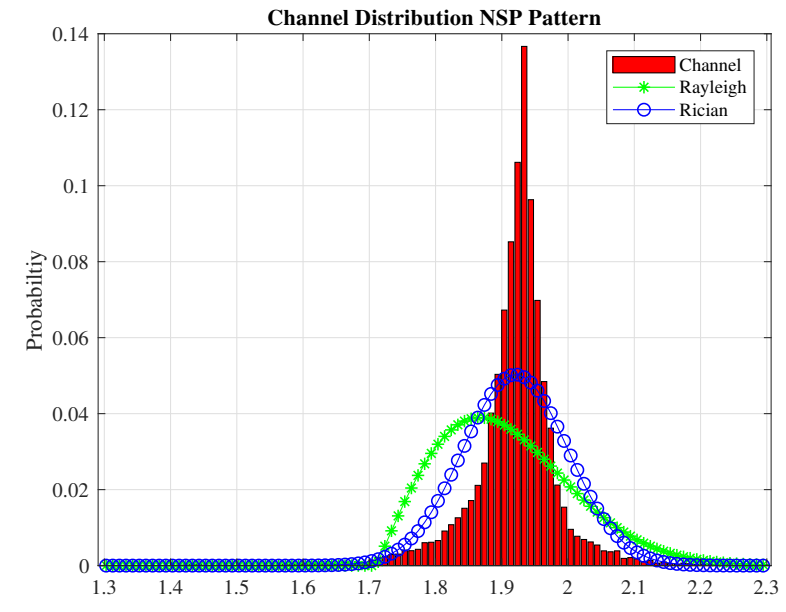

Fig. 9: Channel PDF when NSP is selected.

additional parameter defines the location of the Probability Density Function (PDF) [9].

Figure 9 illustrates channel distribution of SI when NSP is selected, overlapped with candidate PDFs. To quantify the goodness of model fit to statistical data, we calculated the Akaike information criterion (AIC), as well as the Bayesian information criterion (BIC) values and tabulated thye results in Table II. The model with the lowest AIC and BIC is preferred. When NSP is selected, the presence of a strong LOS component centers the distribution away from the origin and keeps it symmetrical in shape. As a result Rician PDF is better fit demonstrating low AIC and BIC (Table II). Rician K-factor is defined as the ratio of signal power in dominant component over the scattered power. For this particular channel, it is estimated to be $24.65 \mathrm{~dB}$, which is another indication of a strong LOS for SI. In the case of SP, there is a significant suppression of SI resulting in skewness and shift towards the origin (Figure 10), where Rayleigh distribution begins to be a better fit as shown in Table II. Rician K-factor in this case is estimated to be $-32 \mathrm{~dB}$ indicating weak LOS and tendency toward Rayleigh distribution.

\begin{tabular}{|c|c|c|c|c|}
\hline Distribution & AIC for NSP & BIC for NSP & AIC for SP & BIC for SP \\
\hline Rayleigh & -43939 & -43922 & -36852 & -36839 \\
Rician & -61358 & -61342 & -35890 & -35879 \\
\hline
\end{tabular}

TABLE II: AIC and BIC values indicating goodness of fit.

\section{CONCLUSION}

This paper presented self-interference channel characterization for MRA based OFDM full-duplex systems. The goal of SI channel measurements is to demonstrate the channel behaviour depending on the achieved suppression levels, system bandwidths and environment dynamics. Comparison of collected SI channel measurements in above mentioned scenarios is performed and significant changes are highlighted. Finally SI channel is statistical modeled by fitting probability distributions to collected channel data and results are compared using goodness of fit test.

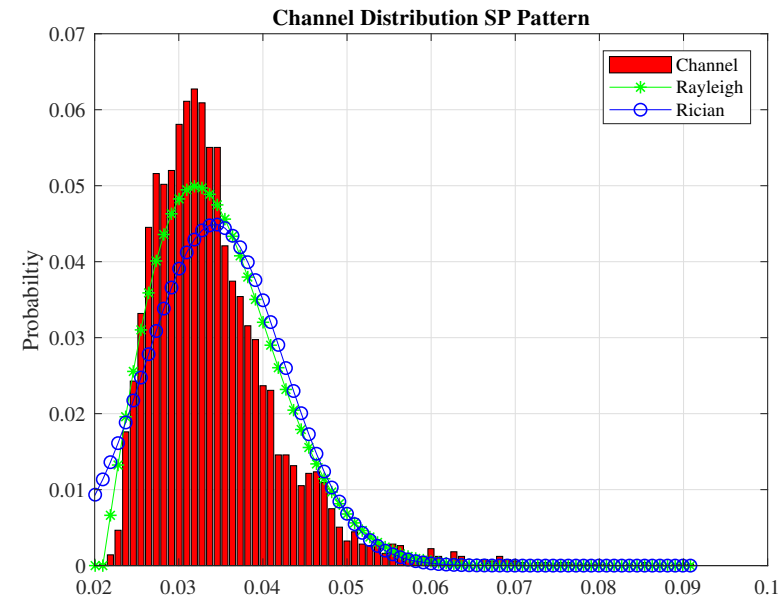

Fig. 10: Channel PDF when SP is selected.

\section{ACKNOWLEDGMENT}

The authors gratefully acknowledge support from the National Science Foundation under award number 1710746.

\section{REFERENCES}

[1] X. Wu, Y. Shen, and Y. Tang, "The power delay profile of the singleantenna full-duplex self-interference channel in indoor environments at $2.6 \mathrm{GHz}$," IEEE Antennas and Wireless Propagation Letters, vol. 13, pp. 1561-1564, 2014.

[2] X. Wu, Y. Shen, and Y. Tang, "Propagation characteristics of the full-duplex self-interference channel for the indoor environment at 2.6 ghz," in 2014 IEEE Antennas and Propagation Society International Symposium (APSURSI), July 2014, pp. 1183-1184.

[3] A. Sethi, V. Tapio, and M. Juntti, "Self-interference channel for full duplex transceivers," IEEE Wireless Communications and Networking Conference, WCNC, vol. 1, pp. 781-785, 2014.

[4] S. N. Venkatasubramanian, L. Laughlin, K. Haneda, and M. A. Beach, "Wideband self-interference channel modelling for an on-frequency repeater," 2016 10th European Conference on Antennas and Propagation, EuCAP 2016, pp. 1-5, 2016.

[5] E. Everett, A. Sahai, and A. Sabharwal, "Passive self-interference suppression for full-duplex infrastructure nodes," IEEE Transactions on Wireless Communications, vol. 13, no. 2, pp. 680-694, 2014.

[6] S. Shaboyan, E. Ahmed, A. S. Behbahani, W. Younis, and A. M. Eltawil, "Frequency and Timing Synchronization for In-Band Full-Duplex OFDM System," in GLOBECOM, 2017.

[7] S. Shaboyan, A. S. Behbahani, and A. M. Eltawil, "Robust Frame Boundary Synchronization for In-Band Full-Duplex OFDM System," Conference Record - Asilomar Conference on Signals, Systems and Computers, vol. 2018-Octob, pp. 1395-1399, 2019.

[8] H. Ju, S. Lee, K. Kwak, E. Oh, and D. Hong, "A new duplex without loss of data rate and utilizing selection diversity," in IEEE Vehicular Technology Conference, no. 1, 2008, pp. 1519-1523.

[9] S. Dey, T. Dey, and D. Kundu, "Two-parameter Rayleigh distribution: Different methods of estimation," American Journal of Mathematical and Management Sciences, vol. 33, no. 1, pp. 55-74, 2014. 